\title{
Total Anthocyanin Content, Total Phenolic Content, and Antioxidant Activity of Various Blueberry Cultivars Grown in Togane, Chiba Prefecture, Japan
}

\author{
Yuma SHIBATA ${ }^{1}$, Kosuke OHARA ${ }^{2}$, Kaori MATSUMOTO ${ }^{2}$, \\ Tetsuya HasegaWA ${ }^{1,2}$ and Masayuki AKIMOTO ${ }^{1,2, *}$ \\ ${ }^{1}$ Graduate School of Pharmaceutical Sciences, and ${ }^{2}$ Faculty of Pharmaceutical Sciences, \\ Josai International University, 1 Gumyo, Togane, Chiba 283-8555, Japan
}

(Received February 13, 2021)

\begin{abstract}
Summary More than fifty cultivated varieties of blueberries are grown under the same processing conditions on the farm at Chiba Prefectural Agricultural College in Japan. The fruits from 51 blueberry cultivars, including 16 rabbiteye (RE) cultivars (Vaccinium ashei Reade) and 35 highbush (HB) cultivars (Vaccinium corybosum L.), were evaluated for total anthocyanin contents, phenolic contents, and their contribution to antioxidant activity among cultivars. Total anthocyanin contents varied from $0.74 \pm 0.21$ ("Barkley") to $4.27 \pm 0.18$ ("Suwannee") $\mathrm{mg}$ as cyanidin-3-glucoside (Cy-3-GC) equivalent/g fresh-weight $(\mathrm{fw})$, with phenolic contents in the range of $0.77 \pm 0.14$ ("Floridablue") to $3.69 \pm 0.89$ ("Suwannee") mg of gallic acid equivalent (GAE)/g fw, which strongly correlated with antioxidant activities assessed using the DPPH and ORAC methods, respectively. Total anthocyanin and phenolic contents were both significantly higher $(p<0.05)$ in RE blueberries than in HB blueberries. Furthermore, the total phenolic values were significantly higher for the RE family than for the HB family $(p<0.01)$. In comparisons of two species, the major anthocyanidin identified were malvidin in RE blueberries and delphinidin in HB blueberries. This result suggests that some RE blueberries, especially "Suwannee," "Homebell" and "Climax," are suitable supply sources with high in vitro antioxidant capacity. This study would be helpful to the quality-oriented cultivation of blueberry.
\end{abstract}

Key Words blueberry, cultivar, anthocyanin contents, phenolic contents, antioxidant activity

The blueberry belongs to the family Ericaceae, subfamily Vaccinoideae, genus Vaccinium, and is native to North American and European regions, in which it is widely cultivated and commercialized (1). This fruit is also popular in markets in Japan as not only a raw food, but also as a supplement and in processed foods. Similar to most berries, this fruit is rich in flavonoids, tannins, and phenolic acids. Previous studies indicated that the blueberry has a number of beneficial health properties due to the presence of bioactive compounds, particularly anthocyanins $(2,3)$. The blueberry is considered to be a health food due to its high antioxidant capacity against free radicals and reactive species and is also one of the greatest sources of antioxidants among all fruits and vegetables $(1,4)$.

Flavonoids have been attracting increasing interest because of their wide variety of physiological functions, including antioxidant activity $(1,2)$. Since blueberries contain high levels of anthocyanins, they have potential as food supplements $(5,6)$. Anthocyanins, which are flavonoids, are the greatest natural pigments giving blue color to plants and exhibit antioxidant potential. This activity may be the main mechanism by which their consumption may reduce the risk of developing

\footnotetext{
*To whom correspondence should be addressed.

E-mail: makimoto@jiu.ac.jp
}

several diseases, such as chronic non-communicable diseases that are stimulated by oxidative processes. The most common anthocyanins present in wild and cultivated blueberries are cyanidin, delphinidin, petunidin and malvidin (7). The antioxidant activity of anthocyanins varies according to their aglycones.

The blueberry includes approximately 400 species in the genus Vaccinium, for example, $V$. ashei and V. corymbosum (1), and anthocyanin contents vary among different varieties. High antioxidant activities in fruits are mainly attributed to phenolic compounds, such as anthocyanins and other flavonoid compounds. Overall antioxidant activity is generally considered to be dependent on the structure and relative content of these compounds in blueberry cultivars. For example, cyanidin has a greater oxygen radical-absorbing capacity than malvidin, peonidin, and petunidin (8). These findings suggest the importance of quantifying individual anthocyanins in blueberry fruits. However, limited information is currently available on the anthocyanin content, phenolic content, and antioxidant activity in each blueberry cultivar grown in Japan, particularly in the Boso region.

Therefore, the objectives of the present study were (i) to quantify total anthocyanins and total phenolic contents in different Japanese blueberry cultivars and (ii) to evaluate their antioxidant activities in order to select 
the richest anthocyanin fraction with the strongest antioxidant activity. The results obtained are discussed with respect to inter- and intraspecies differences in total anthocyanins and the relative antioxidant capacities of these species introduced under the climate conditions of Chiba prefecture in Japan.

\section{MATERIALS AND METHODS}

Chemicals. Cyanidin-3-glucoside (Cy-3-GC) was purchased from Tokiwa Plant Chemistry Co., Ltd. (Chiba, Japan). Trifluoroacetic acid (TFA) was purchased from Kanto Chemicals (Tokyo, Japan) and 6-hydroxy2,5,7,8-tetramethylchroman-2-carboxylic acid (Trolox) and 2,2-diphenyl-1-picrylhydrazyl (DPPH) were obtained Sigma-Aldrich Co., LLC. (St. Louis, MO, USA). Monopotassium phosphate, dipotassium phosphate, and 2-amino-2-hydroxy methyl-1,3-propanediol (Tris) were purchased from FUJIFILM Wako Pure Chemical Corporation (Osaka, Japan). Fluorescein (FL) and 2,2'azobis [2-amino-dipropane] dihydrochloride (AAPH) were purchased from Tokyo Chemical Industry Co., Ltd. (Tokyo, Japan). Other materials and reagents were of the highest commercial grade available.

Plant material. Blueberry fruits were obtained from Chiba Prefectural Agricultural College in Togane, Chiba (N35 $\left.588,292^{\prime} \mathrm{W} 140^{\circ} 379,521^{\prime}\right)$. Fifty-one different genotypes, namely, 35 highbush (HB) and 16 rabbiteye (RE) blueberry cultivars, were sampled and used in the present study. In 2018, fruit samples were harvested from the HB cultivar on June 6th, and the RE cultivar on July 24th. The specific surface areas of the RE and $\mathrm{HB}$ samples were $3.47 \pm 0.41$ and $3.28 \pm 0.51 \mathrm{~cm}^{2} / \mathrm{g}$, respectively. After harvesting, samples were frozen $\left(-30^{\circ} \mathrm{C}\right)$ and immediately stored.

Sample preparation. Anthocyanins were extracted using a modification of a previously reported procedure (9). Three frozen samples were randomly selected and weighed. They were then ground with a mortar and pestle and placed in a screw test tube. Thirty milliliters of $1 \%$ TFA solution was added, and the mixture was placed in a Branson ultrasonic bath (Tosoh, Tokyo, Japan) and then sonicated at $31 \mathrm{kHz}$ for $20 \mathrm{~min}$. After sonication, the mixture was centrifugated at $1,940 \times g$ at $4^{\circ} \mathrm{C}$ for $10 \mathrm{~min}$. After centrifugation, the supernatant was collected and stored in a 100-mL volumetric flask. The residue was re-extracted with $30 \mathrm{~mL}$ of $1 \%$ TFA solution until the supernatant was colorless. Three extractions were performed for each cultivar. The combined supernatant was adjusted to a final volume of $100 \mathrm{~mL}$ and filtered through filter paper. One milliliter of the filtrate was placed in a microtube and centrifugated at $10,000 \times g$ at $4^{\circ} \mathrm{C}$ for $5 \mathrm{~min}$. The extract was then filtered through a $0.45-\mu \mathrm{m}$ membrane filter (GL Sciences, Tokyo) prior to the HPLC analysis.

Determination of total anthocyanins. An analysis of total anthocyanins was conducted using a slight modification of a previously reported procedure (10). HPLC separation conditions were as follows: a Shimadzu HPLC system (LC-10A) equipped with a ODS-HG5 column $(4.6 \times 150 \mathrm{~mm})$ consisting of a DGU-14A degas- ser, LC-10ATvp pump, and CTO-10Avp column oven (Shimadzu, Kyoto, Japan). Anthocyanins were separated by isocratic elution with an aqueous solution of $20 \% \mathrm{CH}_{3} \mathrm{OH}$ containing $0.5 \%$ TFA at a flow rate of $2 \mathrm{~mL} / \mathrm{min}$. The column temperature was maintained at $40^{\circ} \mathrm{C}$. Sample injection was performed using an autosampler (SCL-10Avp, Shimadzu), which loaded $100 \mu \mathrm{L}$ of the sample onto the column. An anthocyanin peak was monitored by absorbance at $520 \mathrm{~nm}$ using an SPD-10Avp UV-VIS detector (Shimadzu). Cy-3-GC was used as the standard product, and each anthocyanin amount was calculated as Cy-3-GC equivalent in compliance with the Italian Pharmacopoeia (11th Edition).

An 8-point external calibration curve composed of Cy-3-GC with concentrations ranging from 0.05 to $30 \mu \mathrm{g} / \mathrm{mL}$. The individual anthocyanin concentrations in the sample solution were calculated from the calibration curve. Data was expressed at $\mathrm{mg} / \mathrm{g}$ fresh-weight (fw). To determine the total content of anthocyanins (T) calculate the content of each anthocyanin (Ti) by mean of the following equation:

$$
\mathrm{Ti}=\frac{(\text { Area }- \text { Int })}{\text { Slope }} \times \underline{Q}
$$

Where Area is the peak area of the individual anthocyanin in the extract; Int, calibration curve intercept; Slope, slope of calibration curve; $Q$, concentration of the fruit mass to be examined in the extract $(\mathrm{g} \mathrm{fw} / \mathrm{mL})$. Data was expressed at $\mathrm{mg} / \mathrm{g}$ fw.

For determination of $T$ by means of the following equation:

$$
T=\Sigma T i
$$

Calculate the percent contribution of anthocyanidins (Pi) among blueberry species means of the following equation:

$$
P i=\frac{\text { Area }_{i}}{\sum \text { Area }_{i}} \times 100
$$

Where $A$ rea $_{\mathrm{i}}$ is the peak area of the individual anthocyanidin and $\Sigma A_{\text {rea }}$ is the peak area of the total anthocyanidins in the sample. Data was expressed at \%.

Assesment of total polyphenolics. The adjusted method with Folin-Ciocalteu reagent (Sigma) was employed to evaluate the total phenolic content (11). The total phenolic content was measured as follows: $0.5 \mathrm{~mL}$ of $80 \%$ ethanol solution was added to $0.5 \mathrm{~mL}$ of the extract. A diluted extract was prepared by adding $0.5 \mathrm{~mL}$ of a $40 \%$ ethanol solution containing $0.5 \%$ TFA to $0.5 \mathrm{~mL}$ of a $40 \%$ ethanol solution of the extract. A standard solution (30-300 $\mathrm{nmol} / \mathrm{mL}$ ) was prepared by adding $0.5 \mathrm{~mL}$ of $1 \%$ TFA to $0.5 \mathrm{~mL}$ of an $80 \%$ gallic acid ethanol solution. Folin-Ciocalteu reagent $(0.25 \mathrm{~mL})$ was added to $0.25 \mathrm{~mL}$ of the diluted extract or standard solution. After allowing the mixture to stand at room temperature for $3 \mathrm{~min}, 0.25 \mathrm{~mL}$ of a $10 \%$ solution of sodium carbonate was added. Then, it was left at room temperature for $60 \mathrm{~min}$, and the absorbance at $750 \mathrm{~nm}$ was measured. A calibration curve was made with standard solutions of gallic acid. The results obtained were expressed as an equivalent of the standard used. All analyses were expressed as mg gallic acid equivalent 
Table 1. Total anthocyanins, total phenolics, and antioxidant activities (DPPH and ORAC) in blueberry fruits of 16 rabbiteye (Vaccinium ashei Reade) cultivars.

\begin{tabular}{lcccc}
\hline & $\begin{array}{c}\text { Total anthocyanins } \\
\text { Cultivar }\end{array}$ & $\begin{array}{c}\text { Total polyphenol } \\
(\mathrm{mg} / \mathrm{g} \text { fw })\end{array}$ & & Antioxidant activity $(\mu \mathrm{mol}$ of TE/g fw $)$ \\
\cline { 4 - 4 } Rabbiteye & & & DPPH & ORAC \\
Suwannee & $4.27 \pm 0.18$ & $3.69 \pm 0.89$ & $29.54 \pm 1.23$ & $83.86 \pm 21.78$ \\
Homebell & $3.96 \pm 0.53$ & $3.62 \pm 0.38$ & $17.85 \pm 0.20$ & $76.91 \pm 10.06$ \\
Climax & $3.88 \pm 0.13$ & $3.40 \pm 0.28$ & $22.44 \pm 0.81$ & $68.39 \pm 9.06$ \\
Southland & $3.47 \pm 0.23$ & $2.30 \pm 0.28$ & $20.35 \pm 0.61$ & $48.94 \pm 9.38$ \\
Ethel & $2.94 \pm 0.56$ & $2.60 \pm 0.24$ & $23.13 \pm 2.69$ & $52.13 \pm 10.90$ \\
Briteblue & $2.80 \pm 0.12$ & $2.07 \pm 0.16$ & $19.31 \pm 0.48$ & $41.37 \pm 3.38$ \\
Menditoo & $2.73 \pm 0.21$ & $2.39 \pm 0.05$ & $16.80 \pm 0.48$ & $60.46 \pm 11.27$ \\
Festival & $2.59 \pm 0.54$ & $1.95 \pm 0.32$ & $19.93 \pm 2.43$ & $40.77 \pm 11.11$ \\
Powderblue & $2.51 \pm 0.31$ & $1.62 \pm 0.73$ & $20.69 \pm 1.69$ & $41.25 \pm 11.96$ \\
Bluegem & $2.49 \pm 0.40$ & $2.11 \pm 0.25$ & $17.28 \pm 1.50$ & $44.63 \pm 8.84$ \\
Woodard & $2.23 \pm 0.48$ & $1.47 \pm 0.44$ & $15.23 \pm 0.38$ & $45.51 \pm 4.39$ \\
Bluebelle & $2.16 \pm 0.41$ & $1.58 \pm 0.81$ & $17.06 \pm 2.67$ & $37.76 \pm 16.18$ \\
Baldwin & $1.92 \pm 0.55$ & $1.82 \pm 0.74$ & $16.12 \pm 2.15$ & $51.88 \pm 6.45$ \\
Nobilis & $1.43 \pm 0.00$ & $1.67 \pm 0.04$ & $12.95 \pm 0.47$ & $38.24 \pm 0.09$ \\
Tifblue & $1.26 \pm 0.33$ & $1.14 \pm 0.22$ & $12.94 \pm 2.70$ & $23.78 \pm 8.43$ \\
Delite & $0.85 \pm 0.38$ & $0.87 \pm 0.18$ & $8.75 \pm 2.31$ & $18.73 \pm 4.16$ \\
Mean & $2.59 \pm 0.98 * * *$ & $2.14 \pm 0.84 * *$ & $18.15 \pm 4.82 *$ & $48.41 \pm 17.37^{* * *}$ \\
\hline
\end{tabular}

Each result shows the mean value and the standard deviation $(n=3)$.

Statistically significant differences: ${ }^{*} p<0.05,{ }^{* *} p<0.01$ and ${ }^{* * *} p<0.001$ compared with HB, respectively.

${ }^{1}$ Mean value of 16 RE blueberry cultivars.

(GAE) per gram of fresh fruits (mg of GAE/g fresh weight of fruit portion, fw).

Antioxidant activity. The in vitro antioxidant activities of blueberry samples were analyzed using two common methods. DPPH and the oxygen radical absorbance capacity (ORAC) assays were implemented by measuring the free-radical scavenging capacities of the extracts relative to that of the standard antioxidant, Trolox. The results of the DPPH and ORAC assays were expressed as Trolox equivalents (TE), namely, the amount of Trolox needed to obtain the same antioxidant capacity of the sample ( $\mu \mathrm{mol}$ of $\mathrm{TE} / \mathrm{g} \mathrm{fw}$ ).

DPPH assay. The DPPH assay is a standard and easy colorimetric method for estimating antioxidant properties by assessing the free-radical scavenging capacities of antioxidants. The scavenging abilities of the samples for DPPH were assessed as previously described with some modifications $(12,13)$. Extracted samples were initially mixed with a $1 \%$ TFA solution and adjusted to a total volume of $1 \mathrm{~mL}$ at various concentrations. The diluted extract $(100 \mu \mathrm{L})$ was mixed with $400 \mu \mathrm{L}$ of $100 \mathrm{mM}$ Tris-HCl buffer (pH 7.4), $250 \mu \mathrm{L}$ of ethanol, and $400 \mu \mathrm{L}$ of DPPH ethanol solution. The control sample was ethanol solution without Trolox and was prepared in the same manner. The mixed solution was stored at room temperature in a dark place and filtered through a $0.45-\mu \mathrm{m}$ membrane filter (GL Sciences) to obtain samples for analysis. The absorbance of the reaction mixture at $517 \mathrm{~nm}$ was recorded using a UV-VIS spectrophotometer (UV-2450, Shimadzu). The true absorbance of the sample was calculated by subtracting the absorbance of the control from that of the measurement sample. The standard curve was created with Trolox, and DPPH radical-scavenging activity was calculated using the following Eq. (4):

Scavenging activity (nmol of $\mathrm{TE} / \mu \mathrm{L}$ of sample solution) $=\mathrm{AS} / \mathrm{AT}$

where AS and AT are the slope of the regression line of sample absorbance and the slope of the regression line of Trolox absorbance, respectively. Antioxidant activity was expressed as nmol Trolox equivalent $\mathrm{g}^{-1}$ fresh weight of fruits ( $\mathrm{nmol}$ of TE/g fw).

ORAC assay. Antioxidant capacity was measured using ORAC assay, as previously described $(14,15)$. FL is decomposed by AOPH-derived ROO', and the process in which fluorescence intensity is attenuated is measured over time. As the decrease in fluorescence intensity becomes slower due to the removal of radicals in the presence of an antioxidant and the antioxidant capacity was evaluated using the AUC (area under the fluorescence intensity-time curve).

TFA solution (1\%) was added to blueberry fruit extracts and diluted 40-, 80-, 100-, and 200-fold to obtain sample solutions. A 20- $\mu$ L Trolox standard, sample, or blank of $75 \mathrm{mM}$ phosphate buffer, $\mathrm{pH} 7.4$ (assay buffer), was added in triplicate to a black-walled 96-well plate. Twenty microliters of the sample solution, Trolox solution or phosphate buffer (blank), and $200 \mu \mathrm{L}$ of 94.4 nM FL in phosphate buffer solution were added to each well to a final concentration of $86 \mathrm{nM}$ per well, and after shaking and stirring, fluorescence intensities ( $f_{0 \text { min }}$ : fluorescence at the start of the record) were measured using a fluorescence plate reader (Cytation 5: Bio- 
Table 2. Total anthocyanins, total phenolics, and antioxidant activities (DPPH and ORAC) in blueberry fruits of 35 highbush (Vaccinum corybosum L.) cultivars.

\begin{tabular}{|c|c|c|c|c|}
\hline \multirow{2}{*}{ Cultivar } & \multirow{2}{*}{$\begin{array}{l}\text { Total anthocyanins } \\
\qquad(\mathrm{mg} / \mathrm{g} \mathrm{fw})\end{array}$} & \multirow{2}{*}{$\begin{array}{l}\text { Total polyphenol } \\
(\mathrm{mg} \text { of GAE/g fw) }\end{array}$} & \multicolumn{2}{|c|}{ Antioxidant activity ( $\mu \mathrm{mol}$ of TE/g fw) } \\
\hline & & & DPPH & ORAC \\
\hline \multicolumn{5}{|l|}{ Highbush } \\
\hline Spartan & $3.36 \pm 0.32$ & $2.38 \pm 0.07$ & $27.74 \pm 2.21$ & $40.36 \pm 3.20$ \\
\hline Lateblue & $2.88 \pm 0.39$ & $2.43 \pm 0.35$ & $20.72 \pm 1.61$ & $51.27 \pm 9.04$ \\
\hline June & $2.55 \pm 0.34$ & $2.29 \pm 0.15$ & $24.94 \pm 1.50$ & $53.29 \pm 1.44$ \\
\hline Burlington & $2.41 \pm 0.50$ & $1.90 \pm 0.18$ & $20.51 \pm 2.06$ & $38.76 \pm 2.43$ \\
\hline Northcountry & $2.41 \pm 0.27$ & $2.43 \pm 0.15$ & $21.08 \pm 1.64$ & $47.04 \pm 12.05$ \\
\hline Concord & $2.21 \pm 0.11$ & $1.76 \pm 0.28$ & $17.67 \pm 0.83$ & $34.33 \pm 5.82$ \\
\hline Elliot & $2.12 \pm 0.55$ & $2.41 \pm 0.17$ & $17.36 \pm 2.27$ & $57.82 \pm 5.81$ \\
\hline Rancocas & $2.05 \pm 0.18$ & $1.75 \pm 0.58$ & $17.41 \pm 0.82$ & $50.32 \pm 2.68$ \\
\hline Bluetta & $2.03 \pm 0.17$ & $1.60 \pm 0.20$ & $16.31 \pm 1.05$ & $30.35 \pm 9.81$ \\
\hline Northland & $1.98 \pm 0.39$ & $2.35 \pm 0.23$ & $19.98 \pm 1.59$ & $41.08 \pm 2.91$ \\
\hline Herbert & $1.84 \pm 0.18$ & $1.55 \pm 0.47$ & $14.71 \pm 1.39$ & $34.94 \pm 12.78$ \\
\hline Earliblue & $1.80 \pm 0.32$ & $2.13 \pm 0.23$ & $18.67 \pm 1.24$ & $41.70 \pm 3.64$ \\
\hline Weymouth & $1.79 \pm 0.15$ & $1.82 \pm 0.21$ & $13.70 \pm 1.19$ & $31.25 \pm 7.48$ \\
\hline Bluejay & $1.75 \pm 0.20$ & $1.16 \pm 0.43$ & $12.63 \pm 0.64$ & $20.26 \pm 8.70$ \\
\hline Goldtraube & $1.71 \pm 0.11$ & $1.97 \pm 0.23$ & $17.13 \pm 1.03$ & $41.30 \pm 4.90$ \\
\hline Northsky & $1.70 \pm 0.09$ & $1.89 \pm 0.27$ & $14.31 \pm 1.47$ & $35.76 \pm 5.71$ \\
\hline Stanley & $1.59 \pm 0.39$ & $1.31 \pm 0.30$ & $17.88 \pm 3.02$ & $40.49 \pm 1.32$ \\
\hline Duke & $1.56 \pm 0.35$ & $1.73 \pm 0.13$ & $14.15 \pm 1.86$ & $32.40 \pm 12.64$ \\
\hline Pemberton & $1.42 \pm 0.07$ & $1.68 \pm 0.18$ & $13.45 \pm 0.69$ & $50.78 \pm 8.63$ \\
\hline Blueray & $1.38 \pm 0.17$ & $1.60 \pm 0.15$ & $11.73 \pm 0.35$ & $31.42 \pm 2.00$ \\
\hline Hitomi & $1.37 \pm 0.16$ & $1.18 \pm 0.28$ & $11.65 \pm 1.64$ & $15.20 \pm 6.37$ \\
\hline Bluecrop & $1.37 \pm 0.16$ & $1.13 \pm 0.16$ & $9.45 \pm 0.91$ & $22.79 \pm 4.86$ \\
\hline Jersey & $1.34 \pm 0.24$ & $1.43 \pm 0.13$ & $10.95 \pm 1.30$ & $31.74 \pm 5.43$ \\
\hline Florida & $1.29 \pm 0.15$ & $1.32 \pm 0.17$ & $12.33 \pm 1.24$ & $31.79 \pm 2.30$ \\
\hline Darrow & $1.19 \pm 0.31$ & $1.40 \pm 0.19$ & $12.06 \pm 1.78$ & $34.97 \pm 10.37$ \\
\hline Dixi & $1.16 \pm 0.07$ & $1.51 \pm 0.25$ & $10.87 \pm 0.18$ & $30.26 \pm 5.61$ \\
\hline Coville & $1.11 \pm 0.10$ & $1.57 \pm 0.09$ & $11.13 \pm 0.98$ & $36.97 \pm 4.19$ \\
\hline Floridablue & $1.10 \pm 0.12$ & $0.77 \pm 0.14$ & $10.19 \pm 0.96$ & $8.30 \pm 3.77$ \\
\hline Sierra & $1.00 \pm 0.18$ & $1.09 \pm 0.32$ & $10.15 \pm 0.79$ & $33.15 \pm 7.43$ \\
\hline Collins & $0.98 \pm 0.02$ & $0.90 \pm 0.49$ & $10.56 \pm 0.82$ & $40.75 \pm 6.46$ \\
\hline Bluehaven & $0.95 \pm 0.16$ & $1.07 \pm 0.28$ & $10.22 \pm 1.63$ & $23.00 \pm 5.98$ \\
\hline Sharpblue & $0.78 \pm 0.06$ & $1.25 \pm 0.22$ & $12.55 \pm 0.84$ & $28.32 \pm 7.23$ \\
\hline Chandler & $0.75 \pm 0.09$ & $1.08 \pm 0.17$ & $6.84 \pm 0.45$ & $24.73 \pm 5.55$ \\
\hline Berkeley & $0.74 \pm 0.21$ & $0.85 \pm 0.06$ & $7.27 \pm 1.37$ & $13.82 \pm 3.92$ \\
\hline Unknown $^{1}$ & $2.58 \pm 0.52$ & $1.40 \pm 0.47$ & $22.98 \pm 3.51$ & $47.35 \pm 2.05$ \\
\hline Mean $^{2}$ & $1.67 \pm 0.63$ & $1.60 \pm 0.48$ & $14.89 \pm 4.99$ & $35.09 \pm 11.44$ \\
\hline
\end{tabular}

Each result shows the mean value and the standard deviation $(n=3)$.

${ }^{1}$ Presumed to be a "Nightgem."

${ }^{2}$ Mean value of $35 \mathrm{HB}$ blueberry cultivars.

Tek, Vermont, USA). Measurements were performed at a fluorescence wavelength of $485 \mathrm{~nm}$, and an excitation wavelength of $520 \mathrm{~nm}$ at $37^{\circ} \mathrm{C}$. Seventy-five microliters of $31.7 \mathrm{mM}$ AAPH in phosphate buffer solution heated to $37^{\circ} \mathrm{C}$ was then added to each well, followed by stirring and shaking. The plate was read from $8 \mathrm{~min}$ to $90 \mathrm{~min}$ at 485 and $582 \mathrm{~nm}$ every $2 \mathrm{~min}$ at $37^{\circ} \mathrm{C}$. AUC was calculated from fluorescence intensity obtained using the following Eq. (5).

$$
\mathrm{AUC}=\frac{f_{8 \min }+f_{10 \min }+f_{12 \min }+\cdots+f_{88 \min }+0.5 \times f_{90 \mathrm{~min}}}{f_{0 \min }} \times 2
$$

Net AUC was the sample or Trolox AUC minus blank AUC. The ORAC value was calculated as the amount of Trolox ( $\mu \mathrm{mol}$ of TE/g) corresponding to $1 \mathrm{~g}$ of blueberry fruit, using the data of samples in which net AUC was within the range of the Trolox calibration curve.

Statistical analysis. Data were expressed as the means of at least 3 independent replicates and were presented as means \pm standard deviations. Statistical analyses were conducted using Tukey's test. Group means were considered to be significant at $p<0.05$ based on minimum significant differences from oneway analysis. Pearson's correlation was used to obtain correlation coefficients ( $R$-values).

\section{RESULTS}

\section{Total anthocyanins}

The total anthocyanin content of each blueberry fruit was estimated as Cy-3-GC equivalent/g fw using the HPLC method. The total anthocyanin content of 

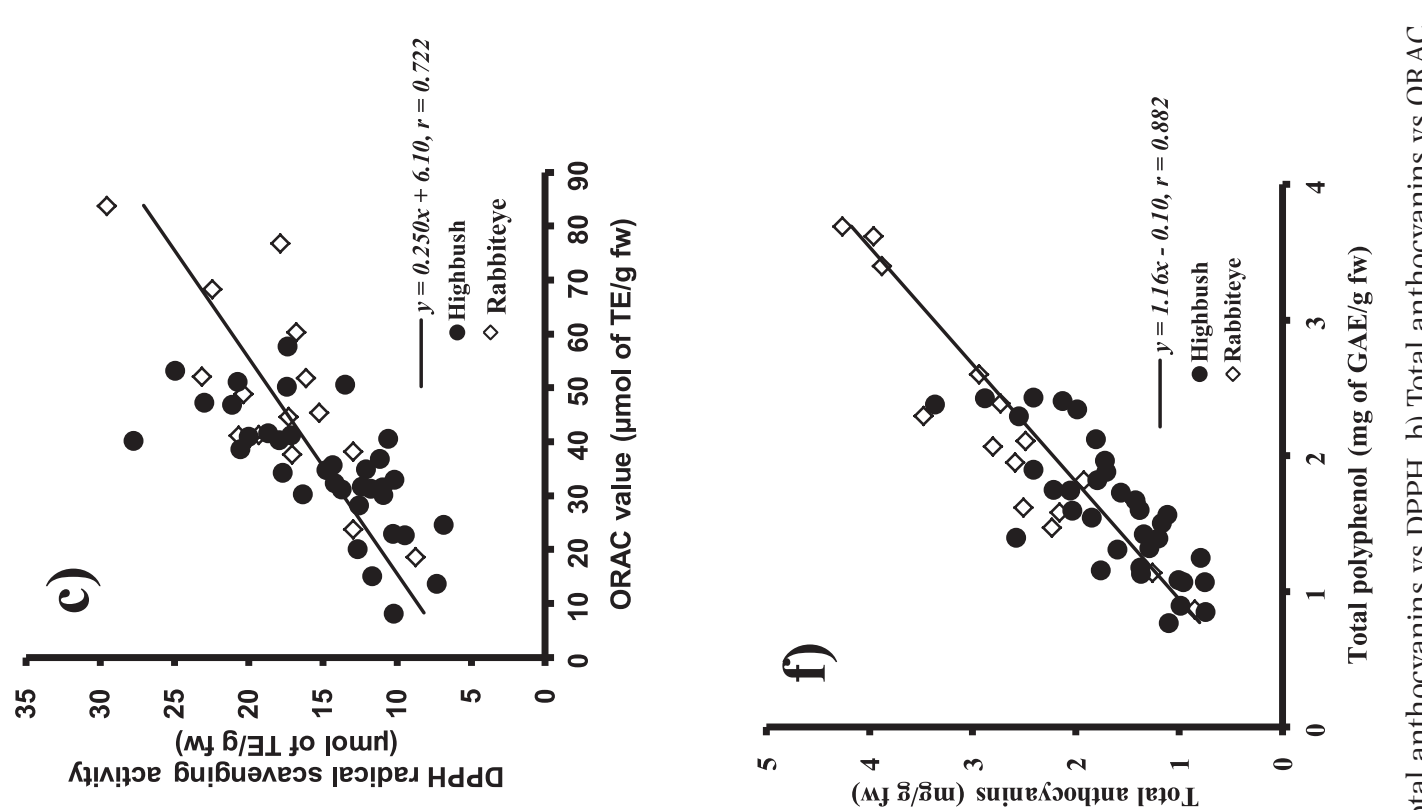

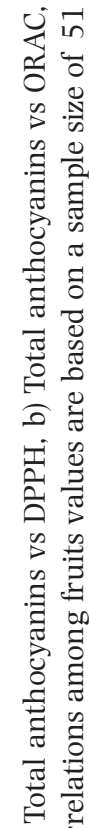

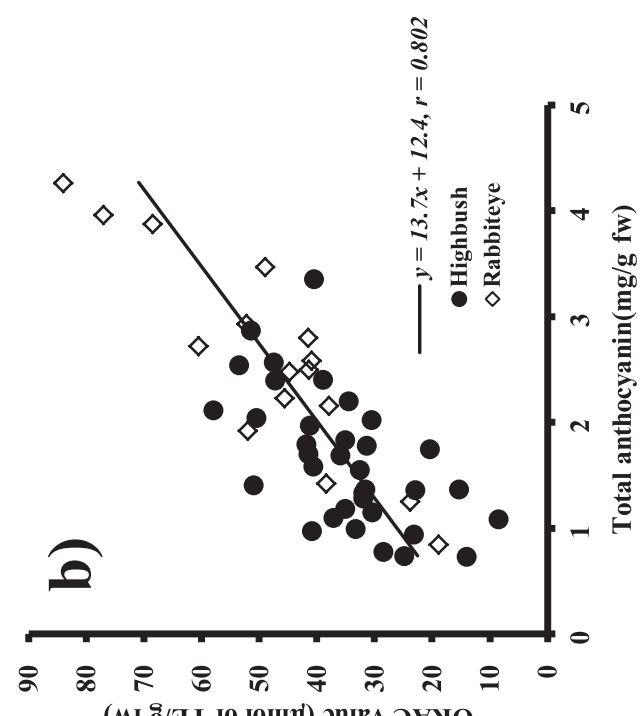

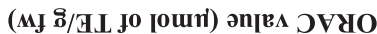

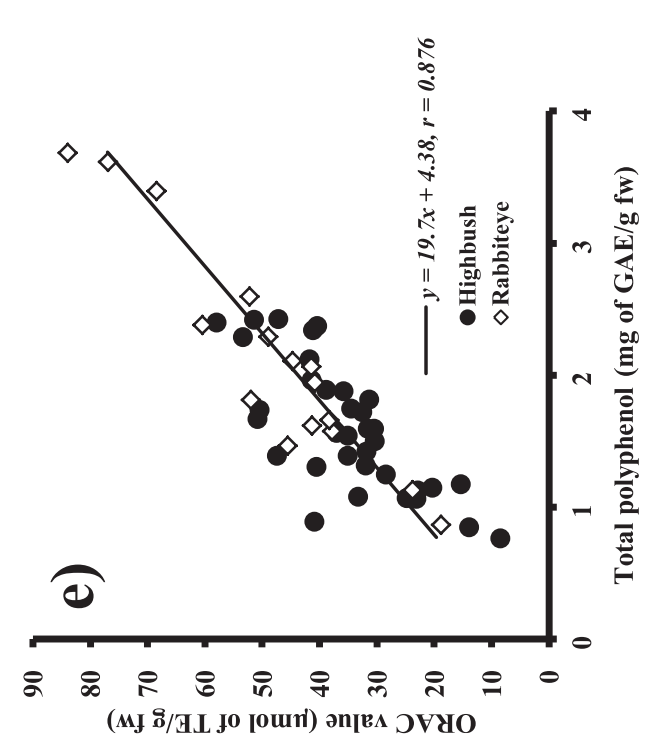

$\dot{s}$

:

त्व

일

旁

$\exists$

㽞

ड़

芩

중

는

ق 양

$\frac{\pi}{8}$

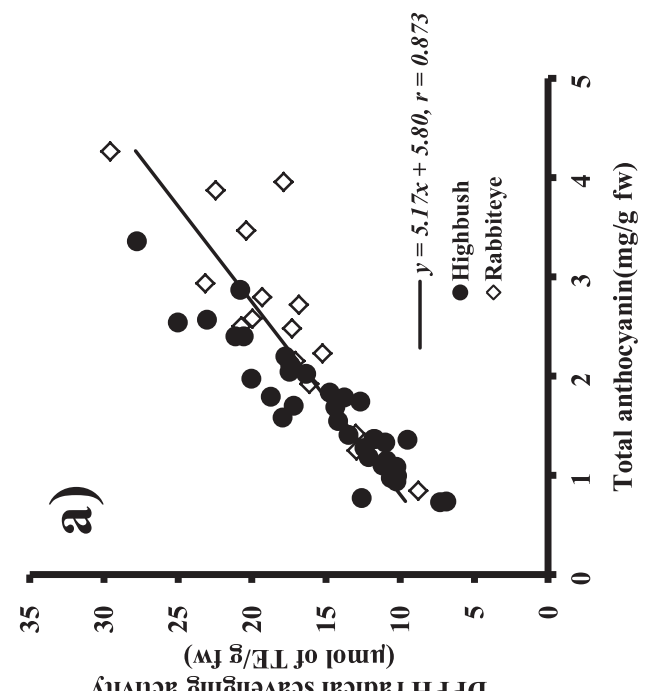

를 苛

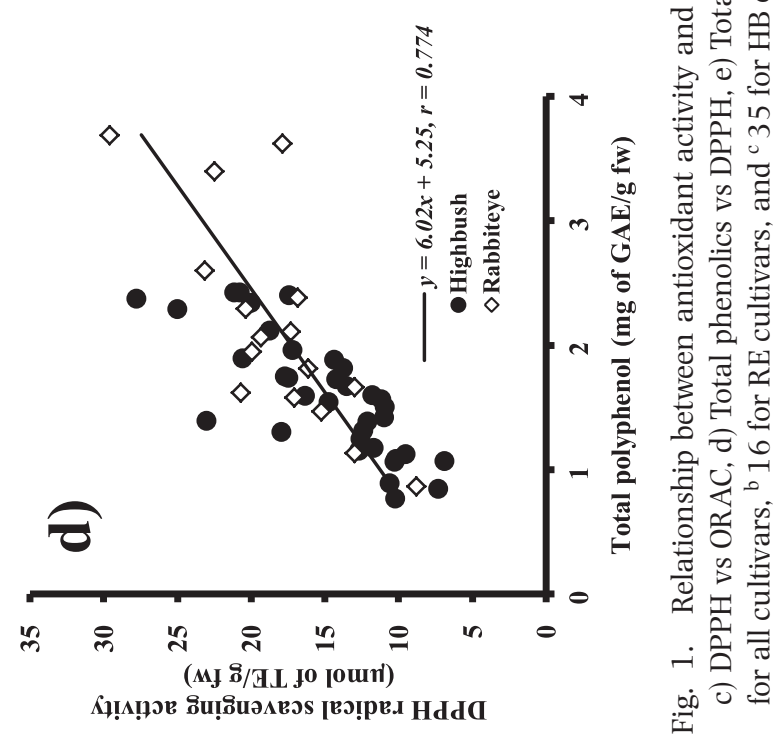


each cultivar is summarized in Tables 1 and 2. An approximately six-fold difference was observed between the lowest ("Berkley"; $0.74 \mathrm{mg}$ total anthocyanin/g fw) and highest concentrations ("Suwannee"; $4.27 \mathrm{mg}$ total anthocyanin/g fw). "Suwannee" had the highest total anthocyanin content followed by "Homebell," "Climax," "Southland," "Spartan," "Ethel" and "Lateblue." The top four varieties "Suwannee," "Homebell," "Climax" and "Southland" all belong to the RE group. In contrast, "Berkley" had the lowest total anthocyanin content in the present study, followed by "Chandler," "Sharpblue," "Sierra," "Blueheaven," and "Collins" (less than $1.0 \mathrm{mg}$ total anthocyanin/g fw). The lowest four varieties all belong to the HB group.

The average total anthocyanin contents of the HB species ( 35 cultivars) and RE species (16 cultivars) were 1.7 and $2.6 \mathrm{mg}$ total anthocyanin/g fw, respectively (Tables 1 and 2). RE blueberries had a markedly higher anthocyanin content (0.85-4.27 mg total anthocyanin/g fw) than HB blueberries (0.74-3.36 mg total anthocyanin/g fw).

Total polyphenolics

Tables 1 and 2 show the amounts of phenolic compounds present in the extracts, expressed as mg of GAE per gram of fresh fruits. Total polyphenols quantified ranged between 3.69 and $0.77 \mathrm{mg}$ of $\mathrm{GAE} / \mathrm{g}$ fw among the 2 groups. In two varieties, the "Suwannee" cultivar contained the highest values, followed by "Homebell" (3.62 mg of GAE/g fw) and "Climax" (3.40 mg of GAE/g fw), while "Floridablue" contained the lowest values.

A 4.8-fold difference was observed between the cultivated sample with the lowest content ("Floridablue") and that with the highest content ("Suwannee") in the present study. A difference was also noted in contents between the HB $(1.60 \pm 0.48 \mathrm{mg}$ of GAE/g fw) and RE (2.14 $\pm 0.84 \mathrm{mg}$ of GAE/g fw) groups. Samples belonging to the RE group had a higher content of phenolic compounds than those belonging to the HB group ( $p<$ 0.01).

Antioxidant activity

The in vitro antioxidant activities of blueberries extracts were analyzed by two common methods, the DPPH and ORAC methods (Tables 1 and 2). The DPPH assay is a standard and easy colorimetric method for estimating antioxidant properties by assessing the free-radical scavenging capacities of antioxidants (Fig. 1a). The scavenging capacities of anthocyanins on DPPH were expressed as TE. A 4.3-fold difference was observed between the cultivated sample with the lowest antioxidant activity ("Chandler"; $6.84 \pm 0.45 \mu \mathrm{mol} \mathrm{TE} / \mathrm{g}$ fw) and that with the highest antioxidant activity ("Suwannee"; 29.54 $1.21 \mu \mathrm{mol}$ TE/g fw). The antioxidant activities (DPPH) of varieties were ranked in the following order: "Suwannee" $>$ "Spartan" $>$ "June" > "Ethel"> "Climax"> "Northcountry"> "Lateblue"> "Powderblue" > "Southland."

The results of the ORAC assay were consistent with those of DPPH assay (Fig. 1b), with some exceptions. A 10-fold difference was noted between the cultivated
Table 3. Percent contribution of anthocyanidins among blueberry species.

\begin{tabular}{lcc}
\hline Anthocyanidin & $\begin{array}{l}\text { Rabbiteye } \\
\text { blueberry }^{1}\end{array}$ & $\begin{array}{c}\text { Highbush } \\
\text { blueberry }\end{array}$ \\
\hline Delphinidin & 16.17 & of total anthocyanidins \\
Cyanidin & $12.93^{* * *}$ & $37.21^{*}$ \\
Petunidin & 16.07 & 8.39 \\
Peonidin & $7.83^{* * *}$ & 20.60 \\
Malvidin & $47.00^{* * *}$ & 2.85 \\
\hline
\end{tabular}

${ }^{1}$ Mean value of 16 blueberry cultivars.

${ }^{2}$ Mean value of 35 blueberry cultivars.

Asterisks in the same column indicate significantly higher percentage: ${ }^{*} p<0.05$ and ${ }^{* * *} p<0.001$.

sample with the lowest antioxidant activity ("Floridablue"; $8.30 \pm 3.77 \mu \mathrm{mol} \mathrm{TE} / \mathrm{g} \mathrm{fw}$ ) and that with the highest antioxidant activity ("Suwannee"; 83.86 $21.78 \mu \mathrm{mol} \mathrm{TE} / \mathrm{g} \mathrm{fw})$. "Homebell" exhibited similar antioxidant activity to "Suwannee." The antioxidant activities (ORAC) of varieties were ranked in the following order: "Suwannee" $>$ "Homebell" $>$ "Climax"> "Menditoo"> "Elliott"> "June"> "Ethel"> "Baldwin"> "Lateblue." In comparisons of blueberry species in the present study, RE blueberries appeared to have a greater scavenging capacity than HB blueberries (Tables 1 and 2).

The relationships between the total anthocyanin content, total phenolic content, and antioxidant activity measured by DPPH and ORAC were investigated in the 51 varieties of blueberries. Antioxidant activity strongly correlated with the total anthocyanin content of 51 blueberries using either assay (DPPH: $r=0.873$, ORAC: $r=0.802)$. In addition, the correlation coefficient of total polyphenolics in blueberry extracts and antioxidant activities measured by the two assays did not markedly differ (DPPH; $r=0.774$, ORAC; $r=0.876$ ). Furthermore, the correlation coefficient of total polyphenols and measured antioxidant activity in the two assays in the blueberry extract did not markedly differ, but was slightly higher using the ORAC method (Fig. 1). Anthocyanin composition

The composition of anthocyanins in the blueberry samples was assessed by a reversed-phase HPLC. The $\mathrm{HB}$ and RE groups had markedly different proportions of anthocyanidins based on the standardized level of anthocyanidins (Table 3). Malvidin was the major anthocyanidin in the RE group, while delphinidin was the major anthocyanidin in the HB group, although its level did not markedly differ from that of malvidin.

\section{DISCUSSION}

Comparisons of the total phenolic content, total anthocyanin content, and antioxidant activity of RE and HB blueberry cultivates have not yet been examined in detail in Japan. The characteristics of each type of cultivar are summarized in Tables 1 and 2. The cultivars with a high concentration of total anthocyanin belonged to the RE species, which is consistent with pre- 
vious findings (16). Total anthocyanin contents were significantly higher in RE blueberry samples than in $\mathrm{HB}$ blueberry samples $(p<0.001)$. Anthocyanin levels in HB blueberry clonal mixtures were approximately 70\% of those in RE blueberries (based on fresh weight). Gao et al. (17) also reported that the total anthocyanin content was higher in RE blueberries produced in southern Brazil than in HB blueberries. In a study by Wang et al. (18), the total amount of anthocyanins estimated from major anthocyanin pigments was higher on average for 36 RE blueberry varieties than for three HB standards. Anthocyanins are only present in the skin of blueberries (19). Previous studies demonstrated that smaller fruits have a higher concentration of anthocyanins because they are more concentrated in the skin than in the pulp $(20,21)$. The specific surface area of RE samples was similar to that of $\mathrm{HB}$ samples $(p=0.284)$. Therefore, total anthocyanin contents may not be attributed to the sizes of the fruits in the present study.

The mean anthocyanin value obtained from $87 \mathrm{HB}$ blueberries by Ehlenfeldt and Prior (20) was very similar to that obtained from the $35 \mathrm{HB}$ blueberries in the present study (1.79 vs $1.70 \mathrm{mg} / \mathrm{g} \mathrm{fw})$. On the other hand, the mean value obtained from our $16 \mathrm{RE}$ cultivars appeared to be higher than those from the 36 RE blueberries by Wang et al. (18). When the findings of these studies were compared with the present results, a quantitative difference was observed among the same blueberry cultivars. For example, the total anthocyanin content of the "Climax" cultivar was $3.88 \mathrm{mg} / \mathrm{g}$ fw in the present study, which was higher than previously reported values of $1.86-2.81 \mathrm{mg} / \mathrm{g}$ fw (18). According to a previous study (11), the biosynthesis of flavonoids increases when plants are grown in bright light or when they are exposed to other types of stress. It may also depend on climatic conditions and the type of cultivation. The total flavonoid and phenolic contents of RE blueberry cultivars grown in Guizhou, China was found to change with ripening stages (22). The amounts of anthocyanins may depend on climatic conditions, different cultivars, the production location, and the year of cultivation $(11,16)$. Therefore, discrepancies between previous findings and the present results may be attributed to differences in variety, maturity, and region.

There were differences between the two species, not only in their total anthocyanin content but also in the ratio of each anthocyanidin. Blueberry fruit contains four major anthocyanidins: delphinidin, cyanidin, petunidin, and malvidin (Table 3). Comparisons of 51 blueberry cultivars revealed that the main anthocyanin pigments were the same; however, there were significant quantitative differences among cultivars. Among RE cultivars, the ranking of anthocyanin types followed the general order of malvidin $>$ delphinidin $>$ petuni$\operatorname{din}>$ cyanidin $>$ peonidin. The proportion of malvidin was greater in RE blueberries than in HB blueberries, which was consistent with previous findings (16). In contrast, the proportion of delphinidin was greater in HB blueberries than in RE blueberries. In addition, the amount of delphinidin was approximately equal to that of malvidin in HB blueberries. A previous study (7) obtained chromatograms representing simple or acylated anthocyanins in HB cultivars, with delphinidin being the major compound. Our results were similar to their findings on the HB cultivar (7). Malvidin is generated by methylation at the $3^{\prime}$ and $5^{\prime}$ positions of delphinidin. Anthocyanin O-methyltransferases (AOMT) might act effectively in the exocarp and endocarp in the RE blueberry. Anthocyanin species composed of delphinidin and petunidin were localized mainly in the exocarp. In contrast, those species composed of cyanidin, peonidin, and malvidin were localized in both the exocarp and the endocarp. These results were inferred to reflect the AOMT activity and the distribution amount of anthocyanidins in the fruit (23). However, a relationship was not observed between individual anthocyanidins and antioxidant activity in plant extracts from blueberries (data not shown).

The total phenolic content in fruit was expressed as GAE in the Folin-Ciocalteu assay. The concentration of total polyphenols in extracts ranged between 0.87 and $3.69 \mathrm{mg}$ of $\mathrm{GAE} / \mathrm{g}$ fw in RE cultivars and between 0.77 and $2.43 \mathrm{mg}$ of $\mathrm{GAE} / \mathrm{g}$ fw in $\mathrm{HB}$ cultivars. Cultivars with the highest total polyphenol contents generally belonged to RE species (Vaccinium ashei Reade), as was the case for total anthocyanin contents. Similar findings were previously reported (16). However, marked differences in total phenolic contents were noted among the same cultivars. According to the results obtained using the same cultivars ("Duke," "Elliot," "Sierra," and "Bluecrop"), total phenolic contents were lower than those reported previously (13). Many factors, such as pre-harvest environmental conditions, the degree of maturity, and growing location, may affect the contents of phenolic compounds (11). A positive correlation ( $r=$ 0.882 ) was observed between total polyphenols and anthocyanins, indicating that the total phenolic content of blueberry cultivars increased as the anthocyanin content became higher. These contents were slightly higher than the corresponding values previously reported (11).

Antioxidant concentrations in extracts are shown in Tables 1 and 2. The ORAC assay measures the scavenging capacity of antioxidants against the peroxyl radical, whereas the DPPH assay is implemented to measure free-radical scavenging capacity (12). It was considered appropriate to combine DPPH based on the electron donation reaction and ORAC, which is an evaluation method based on the hydrogen atom donation reaction, to assess the antioxidant capacity of blueberries in the present study. The values obtained using the DPPH method ranged between 6.84 and $29.54 \mu \mathrm{mol}$ of TE/g fw, whereas those measured by the ORAC method ranged between 8.30 and $83.86 \mu \mathrm{mol}$ of TE/g fw for all cultivars. Previous studies reported the antioxidant activities of fruits as ORAC values $(20,24)$. Comparisons of antioxidant activities obtained by the DPPH method and the ORAC method, it was shown that these values have a relatively similar correlation for blueberry 
cultivars (Fig. 1c). The DPPH method may be a useful alternative to the ORAC method. In the present study, the correlation coefficient for the relationship between the total anthocyanin content and antioxidant activity using the DPPH assay was very high $(r=0.873)$. A strong positive correlation was observed between DPPH and ORAC values $(r=0.722)$, which is consistent with previous findings showing a correlation between total phenolic compounds and antioxidants in plant extracts (24). However, the antioxidant activity measured by the DPPH assay was not always consistent with that measured by the ORAC assay (Table 1). For example, "Homebell," the cultivar with the second highest total anthocyanin concentration and antioxidant activity as measured by the ORAC assay, did not exhibit very strong antioxidant activity by the DPPH assay. ORAC is a robust antioxidant test system that measures hydrophilic and lipophilic chain-breaking antioxidant capacities (14).

Wang et al. (25) examined the antioxidant activity of blueberries and reported that high antioxidant capacity more strongly correlated with its total phenolic content than with its anthocyanin content, and ascorbic acid also made a small contribution to antioxidant activity. Prior et al. (26) also found that the correlation coefficient was much higher between ORAC and the total phenolics $(r=0.85)$ compared to ORAC and anthocyanins $(r=0.77)$. These results are not significantly different from the corresponding values observed in our study. "Suwanee," the cultivar with the highest total phenolic content, also had the highest ORAC values. "Homebell" had the second highest total phenolic contents and ORAC values, followed by "Climax." The correlation of the measured antioxidant activity and total polyphenol content in the ORAC assay $(r=0.876)$ was higher than the oxidation activity measured by the DPPH assay ( $r=0.774)$ (Fig. $1 \mathrm{~d}$ and $1 \mathrm{e}$ ). One explanation is that compounds other than polyphenols are partially responsible for antioxidant activity measured by the ORAC assay. However, these results indicate that approximately $80 \%$ of the antioxidant activity quantified in blueberries was due to the presence of phenolic compounds (13). Varieties with a relatively high total phenolic content had a correspondingly high total anthocyanin concentration, which was consistent with the ORAC value ranking (Fig. 1f).

Fruit maturity at harvest positively affects antioxidant activity and total phenolic and anthocyanin contents, and variations have been reported between different varieties of blueberries. Several other factors influence antioxidant activity, such as production areas, environmental conditions prior to harvest, the stage of ripeness of the fruit after harvesting, and storage conditions $(22,27)$. Therefore, the amounts of phenolic compounds present in blueberries depend not only on the cultivar, but also on production conditions (13). Dried blueberries lost $42 \%$ of the anthocyanins present in fresh fruit (21). In comparisons with synthetic supplements, natural antioxidant activity derived from plant products, such as blueberry fruits, may be more effec- tive at reducing reactive oxygen species due to the synergistic effects of a wide range of constituent biomolecules, including anthocyanins, total phenolics, and vitamin C. Despite the loss of anthocyanin contents, various forms of blueberry nutrients and foods are still good sources of nutrients and bioactive substances. Therefore, the use of blueberry cultivars, which have the richest content of antioxidant substances, will be valuable for the development of new products with beneficial health properties. The blueberry fruit, including rich anthocyanins, have been one of popular consumer products in their fresh form. In addition to the antioxidant effect, many other health effects are associated with blueberries. However, Mazza et al. (2) reported that anthocyanin intestinal absorption is very low at the oral ingested amount. It may be beneficial to take fresh blueberries continuously to maximize their health benefit potential.

The present study markedly expanded the number of blueberry cultivars assayed for polyphenol compositions and antioxidants in fruit tissues and analyzed them under a common set of conditions. The results obtained revealed general trends across cultivars, but specific values should be treated with caution. The values obtained from a single year and location may vary with the year and growing conditions. Nevertheless, the present study revealed a relationship between antioxidant activities and polyphenol contents in blueberry fruit tissues and will be of interest to researchers studying antioxidants and to those attempting to increase antioxidant levels.

\section{CONCLUSION}

The antioxidant activity, total phenolic content, and total anthocyanin content of 51 blueberry cultivars were examined in the present study. Strong correlations were observed between antioxidant activities (ORAC and DPPH) and total anthocyanins and between antioxidant activities and total phenolics. Three of the cultivars ("Suwannee," "Homebell," and "Climax") had the highest total anthocyanin contents, total phenolic contents, and ORAC values. Due to their high total anthocyanin content, total phenolic content, and antioxidant activity, "Suwannee," "Homebell," and "Climax," which belong to the RE blueberry group, have potential nutritional advantages over other cultivars. Based on average values, RE blueberries had higher total anthocyanin contents, total phenolic contents, and antioxidant activity than HB blueberries. Malvidin was the major anthocyanidin in the RE group, while delphinidin was the major anthocyanidin in the $\mathrm{HB}$ group. These results indicate that these blueberry cultivars are a functional food ingredient that is beneficial for human health.

\section{Authorship}

M. Akimoto and Y. Shibata mainly wrote the manuscript. T. Hasegawa and K. Matsumoto designed the research and performed data analyses. K. Ohara and Y. Shibata conducted experiments. 
Disclosure of state of COI

The authors declare that no competing financial interests exist.

\section{Acknowledgments}

We are very grateful to Chiba Prefectural Agricultural College in Japan for providing 51 types of blueberries and Dr. C. Takei for assistance with experiments and analyses. The authors would like to thank Tokiwa Phytochemical Co., Ltd. for their helpful suggestions on the anthocyanin analysis.

\section{REFERENCES}

1) Routray W, Orsat V. 2011. Blueberries and their anthocyanins: Factors affecting biosynthesis and properties. Compr Rev Food Sci Food Saf 10: 303-320.

2) Mazza G, Kay CD, Cottrell T, Holub BJ. 2002. Absorption of anthocyanins from blueberries and serum antioxidant status in human subjects. J Agric Food Chem 50: 7731-7737.

3) Zhou F-H, Deng X-J, Chen Y-Q, Ya F-L, Zhang X-D, Song F, Li D, Yang Y. 2017. Anthocyanin cyanidin-3-glucoside attenuates platelet granule release in mice fed highfat diets. J Nutr Sci Vitaminol 63: 237-243.

4) Drozdz P, Seziene V, Pyrzynska K. 2017. Phytochemical properties and antioxidant activities of extracts from wild blueberries and lingonberries. Plant Foods Hum Nutr 72: 360-364.

5) Johanson MH, de Mejia EG. 2012. Comparison of chemical composition and antioxidant capacity of commercially available blueberry and blackberry wines in Illinois. J Food Sci 71: C141-C148.

6) Ortiz J, Marin-Arroyo M-R, Noriega-Dominguez M-J, Navarro M, Arozarena I. 2013. Color, phenolics, and antioxidant activity of blackberry (Rubus glaucus Benth.), blueberry (Vaccinium floribundum Kunth.), and apple wines from Ecuador. J Food Sci 78: C985C993.

7) Kalt W, McDonald JE, Ricker RD, Lu X. 1999. Anthocyanin content and profile within and among blueberry species. Can J Plant Sci 79: 617-623.

8) Wang H, Cao G, Prior RL. 1997. Oxygen radical absorbing capacity of anthocyanins. J Agric Food Chem 45: 304-309.

9) Shibata Y, Ohara K, Takei C, Matsumoto K, Hasegawa T, Akimoto M, Mitsumoto A. 2018. Influence of measurement method on DPPH free radical-scavenging activity assessment in blueberry fruit extract-Comparison of HPLC method and UV-VIS method-. Josai Kokusai Daigaku Daigakuin Kiyo (The Bulletin of the Graduate School of Josai International University) 22: 167-175 (in Japanese).

10) Ichiyanagi T, Hatano Y, Matsugo S, Konishi T. 2004. Structural dependence of HPLC separation pattern of anthocyanins from bilberry (Vaccinium myrtillus L.). Chem Pharm Bull 52: 628-630.

11) Goncalves C, Guine PF, Teixeira D, Goncalves J. 2015. Evaluation of bioactive phenols in blueberries from different cultivars. Int Scholar Sci Innova 9: 356-359.

12) Tai L, Huang S, Zhao Z, Huang G. 2021. Chemical composition analysis and antioxidant activity of black rice pigment. Chem Biol Drug Des 97: 711-720.

13) Dragovic-Uzelac V, Savic Z, Brala A, Levaj B, Kvacevic
DB, Bisko A. 2010. Evaluation of phenolic content and antioxidant capacity of blueberry cultivars (Vaccinium corymbosum L.) grown in the northwest Croatia. Food Technol Biotechnol 48: 214-221.

14) Huang D, Ou B, Hampsch-Woodill M, Flanagan JA, Prior RL. 2002. High-throughput assay of oxygen radical absorbance capacity (ORAC) using a multichannel liquid handling system coupled with a microplate fluorescence reader in 96-well format. J Agric Food Chem 50: 4437-4444.

15) Oki T. 2007. Ministry of Agriculture, Forestry and Fisheries Subsidy Project, Food Functionality Evaluation Manual Collection II (revised 2nd edition), p 71-86.

16) Pertuzatti PB, Barcia MT, Rodrigues D, da Cruz PN, Hermosin-Gutierrez I, Smith R, Godoy HT. 2014. Antioxidant activity of hydrophilic and lipophilic extracts of Brazilian blueberries. Food Chem 164: 81-88.

17) Gao L, Mazza G. 1994. Quantitation and distribution of simple and acylated anthocyanins and other phenolics in blueberries. J Food Sci 59: 1057-1059.

18) Wang SY, Chen H, Camp MJ, Ehlenfeldt MK. 2012. Flavonoid constituents and their contribution to antioxidant activity in cultivars and hybrids of rabbiteye blueberry (Vaccinium ashei Reade). Food Chem 132: 855864.

19) Lee J, Wrolstad RE. 2004. Extraction of anthocyanins and polyphenolics from blueberry processing waste. $J$ Food Sci 69: 564-573.

20) Ehlenfeldt M, Prior RL. 2001. Oxygen radical absorbance capacity (ORAC) and phenolic and anthocyanin concentrations in fruit and leaf tissues of highbush blueberry. J Agric Food Chem 49: 2222-2227.

21) Reque PM, Steffens RS, Martins da Silvia A, Jagblonski A, Flores SH, Rios AO, Vogt de Jong E. 2014. Characterization of blueberry fruits (Vaccinium spp.) and derived products. Food Sci Technol 34: 773-779.

22) Xie G, Xu X, Zhou X, Liu Y, Zhao Z. 2019. Changes in phenolic profiles and antioxidant activity in rabbiteye blueberries during ripening. Int J Food Proper 22: 320329.

23) Yoshimura Y, Enomoto H, Moriyama T, Kawamura Y, Setou M, Zaima N. 2012. Visualization of anthocyanin species in rabbiteye blueberry Vaccinum ashei by matrix-assisted laser desorption/ionization imaging mass spectrometry. Anal Bioanal Chem 403: 18851895.

24) Dudonne S, Vitrac X, Coutiere P, Woillez M, Merillon J-M. 2009. Comparative study of antioxidant properties and total phenolic content of 30 plant extracts of industrial interest using DPPH, ABTS, FRAP, SOD, and ORAC assays. J Agric Food Chem 57: 1768-1774.

25) Wang H, Cao G, Prior RL. 1996. Total antioxidant capacity of fruits. J Agric Food Chem 44: 701-705.

26) Prior RL, Cao G, Martin A, Sofic E, McEwen J, O’Brien C, Lischner N, Ehlenfeldt M, Kalt W, Krewer G, Mainland CM. 1998. Antioxidant capacity as influenced by total phenolic and anthocyanin content, maturity, and variety of Vaccinium species. J Agric Food Chem 46: $2686-$ 2693.

27) Connor AM, Luby JJ, Hancock JF, Beckheimer S, Hanson EJ. 2002. Changes in fruit antioxidant activity among blueberry cultivars during cold-temperature storage. J Agric Food Chem 50: 893-898. 